\title{
Actividad física de adolescentes: implicación de sustancias nocivas, modalidad practicada y familia
}

\author{
José Ignacio Álvaro González \\ Universidad de Granada - Granada - España \\ Félix Zurita Ortega \\ Universidad de Granada - Granada - España \\ Virginia Viciana Garófano \\ Universidad de Granada - Granada - España \\ Asunción Martínez Martínez \\ Universidad de Granada - Granada - España \\ Susana García Sánchez \\ Centro Educativo Jabalcon - Baza - Granada - España \\ Manuel Estévez Díaz \\ Universidad de Granada - Granada - España
}

\section{Resumen}

Los objetivos de la investigación fueron: determinar los niveles de práctica de actividad física en adolescentes, consumo de tabaco y alcohol como fenómeno social, establecer qué tipo de actividad y nivel federativo y dictaminar el nivel de AF en los progenitores; y apreciar cómo influye en la práctica de actividad física regular, el consumo de sustancias, el tipo de deporte practicado y el nivel de práctica de padres y madres. Participaronun total de 2.134 adolescentes, empleándose una hoja de autoregistro para las variables: Práctica de AF, Modalidad, Estado Federativo y Nivel de Actividad Física Familiar; el FTND para determinar el Consumo de Tabacoy el AUDIT para elConsumo de Alcohol. Los principales resultados indican que los adolescentes que practican actividad física no consumen habitualmente sustancias nocivas, están influenciados por parámetros familiares, realizan en mayor medida deportes de tipo colectivo y están federados en ellos.

Palabras clave: Actividad Física; drogas; familia.

Physical activity in adolescents: involvement of harmful substances, and practiced family mode

\begin{abstract}
The objectives of the research were: to determine the levels of physical activity in adolescents, consumption of snuff and alcohol as a social phenomenon, establish what type of activity and federal level and determine the level of PA in parents, and see how it affects the practice of physical activity regular substance use, the type of sport and level of practice of parents.A total of 2,134 adolescents, using a sheet of selfregistration for the variables: Practice AF mode, and federal state level Family Physical Activity, the FTND to determine Snuff consumption and AUDIT for Alcohol Consumption. The main results indicate that adolescents who practice physical activity does not usually consume harmful substances, parameters are influenced by family, made further collective sports type and are federated in them.
\end{abstract}

Keywords: Physical Activity; drugs; family.

\section{Atividade física por adolescentes: implicação de substâncias nocivas, modalidade praticada e família}

\section{Resumo}

Os objetivos da investigação foram: determinar os níveis de prática de atividade física em adolescentes, o consumo de tabaco e álcool como fenômeno social, estabelecer o tipo de atividade e nível federativo e determinar o nível de AF nos progenitores; verificar a influência da prática regular de atividade física, o consumo de substâncias, o tipo de esporte praticado e o nível de prática de pais e mães. Participaram 2.134 adolescentes, utilizando-se uma folha de autorregistro para as variáveis: Prática de AF, Modalidade, Estado Federativo e Nível de Atividade Física Familiar; o FTND para determinar o Consumo de Tabaco e o AUDIT para o Consumo de Álcool. Os principais resultados indicam que os adolescentes que praticam atividade física habitualmente não consomem substâncias nocivas, estão influenciados por parâmetros familiares, realizam mais esportes de tipo coletivo e estão federados neles.

Palavras-chave: Atividade Física; drogas; família. 


\section{Introducción}

El alcohol y el tabaco se conforman como dos de las sustancias perjudiciales para el organismo más consumidas en nuestra población, considerándose entre los principales problemas de salud en España. En 2011, según los datos obtenidos por el Plan Nacional sobre Drogas (2013), un $76,6 \%$ de la población en España ha consumido alcohol en alguna ocasión y un $40,2 \%$ ha fumado. Dichas cifras se tornan aún más preocupantes al saber que el consumo se inicia en la etapa adolescente (15-17 años), existiendo un $62,2 \%$ que ha bebido alcohol en alguna ocasión a lo largo de su vida y un $26,6 \%$ tabaco. Debido a las repercusiones negativas que ello acarrea, los científicos sociales y de la salud se centran en el análisis de esta situación (Villarreal-González, Sánchez-Sosa, Musitu,\& Varela, 2012).

Sobradamente conocidas son las repercusiones físicas y fisiológicas que ocasiona el consumo de alcohol y tabaco en el cuerpo humano (Maurage, Joassin, Speth, Modave, Philippot, \& Campanella, 2012). No menos importancia se le debe dar a la interrupción que el alcohol puede producir en el avance de ciertas capacidades básicas adquiridas en la etapa pubescente, como son el pensamiento abstracto y la metacognición (Cortés, Espejo, Giménez, Luque, Gómez,\& Motos, 2011).

La etapa adolescente es crítica en la aparición del hábito social de la toma o ingesta de alcohol (Giró, 2007). Dicha fase, se considera una transición entre la infancia y la adultez, caracterizando al adolescente por tener sus propios valores, hábitos, reglas, espacios y modas (Villarreal-González, Sánchez, \& Musitu, 2013). En estas edades se produce un desarrollo esencial en la persona, debido a que se acatan normas del grupo de iguales al que se pertenece, se aceptan compromisos e interiorizan valores, configurándose de este modo el ámbito cognitivo, físico y social (Woolfolk, 2008; Carballo \& cols., 2013). Por tales circunstancias, es muy importante que se adquieran hábitos saludables y se inhiba el consumo de sustancias nocivas, ya que las probabilidades de seguir los mismos patrones de conducta en la etapa adulta son elevadas, incluso existe un mayor riesgo de medrar en su abuso (Ruiz-Ruisueño, Ruiz-Juan, \& Zamarripa, 2011).

En este sentido, es imprescindible controlar los factores de riesgo que más fuerza ejercen en la adquisición de hábitos sobre el adolescente. Nebot, Tomás, Ariza, Valmayor, López y Juárez (2004), señalan que los factores influyentes en el inicio de consumo de alcohol y tabaco, provienen del ámbito socioeconómico, cognitivo, emocional y familiar (Pons, 1998). Las costumbres alcanzadas por familiares así como por personas del entorno próximo al joven, inciden para afianzar, apoyar o suprimir su patrón de conducta (Londoño, 2010; Ruiz-Juan \& Ruiz-Ruisueño, 2011).

Como vemos, son múltiples los factores de riesgo en el inicio del consumo de alcohol y tabaco por parte de los adolescentes, pero múltiples estudios tachan el ámbito familiar como el más influyente. En este sentido, es trascendental los modelos de interacción utilizados, la unión, la ductilidad de los miembros de la familia y el consumo de los propios padres (Musitu \& Pons, 2010).

Una comunicación familiar adecuada y positiva, contribuye a mejorar la adaptabilidad y cohesión familiar; por el contrario, cuando el trato no es adecuado existe mayor probabilidad de consumir alcohol y drogas por parte de los hijos (Cava, Murgui, \& Musitu, 2008). A colación de esto, Elzo (2010), observó que los adolescentes que abusaban en el consumo de sustancias nocivas, percibían como negativa la relación familiar, con un alto grado de conflictos y bajo entendimiento entre sus componentes.

Otro factor a considerar en el sector familiar es el hábito adquirido por los padres, habiendo más posibilidad de consumo por los hijos cuando sus padres toman bebidas alcohólicas y fuman (Jiménez, Musitu, \& Murgui, 2008). Así, cuando un adolescente percibe el consumo de alcohol y tabaco por parte de sus padres como un acto encuadrado dentro de la normalidad, lo extrapolará como tal cuando se relacione socialmente con sus amigos.

Muy vinculado al riesgo familiar, y no menos importante, se encuentra el grupo de iguales al adolescente, donde se establecen diversos roles en función de la agrupación a la que se pertenezca, potenciando ciertos comportamientos, estando presente, entre otros, el consumo de alcohol y tabaco (Henry, Slater, \& Oetting, 2005).

El ámbito cognitivo significa otra gran amenaza, ya que los adolescentes sospechan que la ingesta de alcohol y tabaco es una acción que otorga mayor seguridad y serenidad, así como una vía para relacionarse con su grupo de amigos (Ariza, Nebot, Villalbí, Díez, Tomás, \& Valmayor, 2003). A su vez, interpretan como positivas las impresiones relacionadas a la inhalación de nicotina, como la sensación de confort o como variable para controlar en peso, aspecto en el que se centran bastante las chicas en estas edades (Nerín, Beamonte, Gargallo, Jiménez-Muro, \& Marqueta, 2007).

Tal y como afirman Buelga, Musitu y Murgui (2009), por todas estas razones y muchas más, es muy importante concederle mayor importancia a las agrupaciones de condición voluntaria como parroquias, grupos de ayuda mutua etc., con objeto de reforzar la identidad personal y el autoestima en la etapa adolescente.En esta línea, se debe ejercer una labor coherente y orientada hacia la consecución de hábitos adecuados para la salud, donde el consumo de sustancias nocivas y drogas sea mínimo. Una buena alternativa para lograr tal fin, es encaminarlos hacia la práctica de actividad física desde edades tempranas, lo cual repercute positivamente en el estado de salud del individuo así como su inclusión en núcleos de vida saludable (Krekoukia, Nassis, Psarra, Skenderi, Chrousos, \& Sidosis, 2007).

La Organización Mundial de la Salud (2010) aconseja que los niños en edades comprendidas entre los 5 y 17 años, debieran practicar al menos 60 minutos diarios de actividad física moderada o vigorosa. Son variados los estudios y resultados que se han encontrado en cuanto a la investigación de los niveles de actividad física en adolescentes, como el llevado a cabo por Oviedo y cols. (2013), en Roses (Girona) donde el $45,5 \%$ de los varones y el $14,84 \%$ de las mujeres 
cumplen las recomendaciones anteriormente comentadas. Peores resultados hallaron Abarca-Sos, Zaragoza, Generelo y Julián (2010) en Huesca, donde sólo el 7,54\% de los chicos y el $3,44 \%$ de las chicas lo llevaban a cabo. Por el contrario, en Madrid la práctica de Actividad Física es mayor, representando el un $82,2 \%$ los chicos y un $60,7 \%$ las chicas (Martínez-Gómez, Welk, Calle, Marcos, \& Veiga, 2009).

Como podemos observar, en líneas generales, la práctica de actividad física por parte de los adolescentes no cumple las recomendaciones de la OMS. Se pone de manifiesto que los varones practican más actividad física que las mujeres, lo cual nos hace pensar que éstos sienten más atracción por las actividades deportivas ofertadas en los municipios y centros escolares que las chicas. Debido a ello, es importante investigar cuáles son las actividades deportivas que más seducen a los adolescentes y actuar con base en ello, con objeto de incrementar el número de personas que realicen práctica de actividad física.

Diversos son los estudios que han centrado su atención en esta línea, como el llevado a cabo por Luengo (2007) con alumnos de Primaria de Carabanchel de Madrid, donde el deporte más practicado era el balonmano $(21,9 \%)$ seguido del fútbol y artes marciales $(16,4 \%)$ y baile (10,9\%). Por otro lado, Ruiz, García, y Hernández (2001) afirman que un $44,8 \%$ del alumnado almeriense de Educación Secundaria post-obligatoria, se decanta por la práctica de deportes colectivos, frente a un $33,7 \%$ que prefiere modalidades individuales. El 4,8\% restante practica actividades físico-deportivas no regladas como pasear, correr, patinar etc.

Uno de los estudios más completos al respecto es el de García (2006), el cual exploró cuáles eran los deportes más practicados en la población española desde 1980 hasta 2005 , siendo la natación (recreativa) la más experimentada con un $33 \%$. Le sigue el fútbol con un $31,7 \%$ y el ciclismo con un $19,1 \%$. Basándonos en éste último estudio, podemos comprobar cómo es posible alcanzar objetivos y conseguir cambios, como lo ocurrido con la práctica de un deporte apenas ejercido hace algunas décadas como es la natación. La construcción de piscinas climatizadas y la oferta por parte de los municipios de actividades acuáticas, tienen gran parte de culpa en este sentido.

Por este motivo, el primer objetivo del presente estudio es determinar los niveles de práctica de actividad física en adolescentes, el consumo de tabaco y alcohol como fenómeno social, establecer qué tipo de actividad y nivel federativo o dictaminar el nivel de AF en los progenitores; y un segundo objetivo para apreciar cómo influye en la práctica de actividad física regular, el consumo de sustancias, el tipo de deporte practicado y el nivel de práctica de padres y madres.

Según estos objetivos se plantean tres hipótesis:

- Los chicos y chicas que ingieran de forma habitual alcohol y tabaco no realizaran AF de forma regular.

- La práctica de deportes colectivos fomentara la realización de AF de forma regular.
- A mayor práctica de los progenitores aumenta la realización en los adolescentes.

Este estudio nos reporta datos novedosos sobre el perfil de adolescentes practicantes habituales de AF y sobre como intervienen en él factores sociales, deportivos y académicos.

\section{Material Y métodos}

\section{Participantes}

En este trabajo de carácter observacional, descriptivo y de tipo transversal participaron un total de 2.134 adolescentes españoles, de ambos sexos (49,8\% de hombres y $50,2 \%$ de mujeres), de 15 a 18 años (M=15,93 años; DT= $\mathbf{0}, 853)$, de 20 centros educativos de distintas zonas de la provincia de Granada y distribuidos en el Segundo Ciclo de la Educación Secundaria Obligatoria (ESO). La muestra es representativa en cuanto al número de alumnos/as analizados que se encuentran matriculados en Segundo Ciclo de Educación Secundaria Obligatoria en la provincia de Granada ( $n=18.930$ ), asumiendo un error muestral del0.02, con un nivel de confianza del $95 \%$, la muestra requerida fue de 2.231 participantes. Para seleccionar los participantes se establece un muestreo aleatorio por conglomerados como indican Santos, Muñoz, Juez y Cortiñas (2003).

\section{Variables e instrumentos}

En este estudio de investigación se tomó como referencia las siguientes variables e instrumentos:

- Escala de Consumo de Tabaco, se recoge del cuestionario original "Fagerström Test for Nicotine Dependence (FTND)" de Heatherton, Kozlowski, Frecker, \& Fagerström (1991), y traducido al castellano por Villareal- González (2009), establece el número o cantidad de cigarros, el impulso por fumar y la dependencia de nicotina que se tiene. Este test consta de 6 preguntas, de las cuales las 4 primeras son de tipo dicotómico $(0=\mathrm{No}$ y $1=\mathrm{Si})$, y los dos ítems restantes se valoran con una escala Likert de cuatro opciones, donde el 0 es Nunca y el 3 es Siempre; se establece la suma que oscila entre el 0 y el 10 , y se establecen cuatro categorías $(0=$ No fumador, de 1 a $4=$ Dependencia Baja, 5 a $6=$ Dependencia Moderada y $>6=$ Dependencia Alta). En el estudio de Villareal-González (2009) se determinó una fiabilidad de $\alpha=.970$ similar a la que se detecta en nuestro trabajo $\alpha=.956$.

- Escala del Consumo de Alcohol, procede del cuestionario original "Alcohol Use Disorders Identification Test (AUDIT)" de Saunders, Aasland, Babor, De la Fuente y Grant (1993), y adaptado al 
español por Rubio (1998), consta de 10 preguntas, donde las 8 primeras cuestiones tienen cinco posibles respuestas donde el 0 es Nunca y el 4 es a Diario o Mas, y los dos ítems restantes se valoran con tres posibles opciones que puntúan 0-2 y 4 ; se establece la suma que variará entre el 0 y el 40 , y señalar que las tres primeras preguntas hablan de la cantidad y frecuencia de consumo de alcohol, las preguntas 4-5 y 6 determinan la dependencia de consumo de alcohol y los ítems del 7 al 10 reportan consumo dañino o perjudicial. En el estudio de Rubio (1998) se determinó una fiabilidad de $\alpha=.800$ similar a la que se detecta en nuestro trabajo $\alpha=.842$.

- Práctica de Actividad Física, con la opción dicotómica de si práctica o no, utilizándose una hoja de registro para ello, donde se instaba a marcar la opción de $\mathbf{S i}$, siempre y cuando el adolescente realizase Actividad Física fuera del Centro Escolar con una frecuencia igual o superior a tres horas semanales y No, si la frecuencia era inferior a 3 horas semanales.

- Modalidad y tipo de Actividad Física, con cinco opciones de respuesta, empleándose para ello una hoja de autorregistro, donde se planteaban cinco modalidades cada una de ellas con ejemplos para evitar errores de apreciación, de forma que las categorías planteadas eran: a) No Practica, b) Deporte Individual (tenis, atletismo, natación, escalada, ciclismo, ...), c) Deporte Colectivo (futbol, baloncesto, balonmano, voleibol, rugby, waterpolo,....), d) Actividades Gimnasio (artes marciales, spinning, pesas, aerobic,....) y e) Otros (realizan actividades variadas pero no con regularidad).

- Federado, con la opción dicotómica de Si está federado o No, instando al adolescente a responder a la cuestión en referencia a los dos últimos años.

- Nivel de Actividad Física Familiar, dividida en dos opciones, la opción de Si, siempre y cuando uno de los dos progenitores realizase Actividad Física con una frecuencia igual o superior a tres horas semanales y No, si la frecuencia era inferior a 3 horas semanales.

\section{Procedimiento}

En primer lugar, a través de la Facultad de Ciencias de la Educación de la Universidad de Granada (Área de Corporal), y en contacto con la Delegación de Educación de la Junta de Andalucía, se solicitó la colaboración de los Centros Educativos de la Provincia de Granada seleccionados, a partir de un muestreo de conveniencia de las categorías objeto de estudio. La dirección de cada centro educativo fue informada sobre la naturaleza de la investigación y solicitando la colaboración de sus alumnos/as. En segundo lugar se adjuntó un modelo de autorización destinado a los responsables legales de los adolescentes pidiéndoles su consentimiento informado, garantizando en todo momento a los participantes el anonimato de la información recogida aclarando que su utilización sería sólo con fines científicos. Los encuestadores estuvieron presentes durante la recogida de los datos que se llevó a cabo en sus aulas habituales durante un periodo regular de clase, desarrollándose sin ningún tipo de problema o anormalidad a reseñar. En último lugar se agradeció a los profesores y responsables su colaboración y se les informó del envío en un futuro próximo de un informe sobre los datos obtenidos respetando la confidencialidad de los mismos.El estudio cumplió con las normas éticas del Comité de Investigación y Declaración de Helsinki de 1975. Se contó en todos los casos con el consentimiento informado de los participantes y se respetó el resguardo a la confidencialidad.

\section{Análisis de los Datos}

Para el análisis de los datos se utilizó el programa estadístico software SPSS 20.0., mediante la utilización de diversas técnicas de análisis, de esta forma se establecieron los descriptivos básicos mediante la utilización de medias y frecuencias y para el estudio relacional se emplearon las tablas de contingencia.

\section{Resultados}

En cuanto a los resultados obtenidos en primer lugar observamos en la primera tabla (Tabla I), que los 2.134 adolescentes presentaban proporciones similares por género, el $90,9 \%(n=1.939)$ eran no fumadores, respecto al consumo de alcohol, la cantidad y frecuencia era escasa o no consumían en un $70,3 \%$ de los jóvenes, que el consumo medio era perceptible en un $23,1 \%$ y los que abusaban del mismo solo se reflejaba en un $6,6 \%$ de los casos; en cuanto a la dependencia el $79,4 \%$ no tenían ningún tipo se asociación con las bebidas alcohólicas y en la misma línea el $65,3 \%$ señaló no provocar ningún daño tras la ingesta de la misma mientras que el restante $34,7 \%$ declaró mostrar actitudes dañinas tras la ingesta de estos productos.

En cuanto ala práctica regular de Actividad Física el $67,1 \%$ indicaban hacerla de forma habitual, de estos la mayoría se inclinaban por desarrollar deportes colectivos y actividades físicas variadas (otros), asimismo un $21,9 \%$ de los adolescentes se encontraban federados en sus especialidad deportiva y la mitad de los jóvenes señalaban que al menos alguno de sus padres realizaba actividad física de manera habitual durante la semana. 
Tabla I. Descriptivos de las variables.

\begin{tabular}{|c|c|c|}
\hline \multicolumn{3}{|c|}{ Género } \\
\hline \multicolumn{2}{|c|}{ Masculino } & $49,8 \%(n=1.062)$ \\
\hline \multicolumn{2}{|c|}{ Femenino } & $50,2 \%(n=1.072)$ \\
\hline \multicolumn{3}{|c|}{ Consumo Tabaco (FTND) } \\
\hline \multicolumn{2}{|c|}{ No Fumador } & $90,9 \%(n=1.939)$ \\
\hline \multicolumn{2}{|c|}{ Dependencia Baja } & $7,4 \%(n=158)$ \\
\hline \multicolumn{2}{|c|}{ Dependencia Media } & $0,9 \%(n=19)$ \\
\hline \multicolumn{2}{|c|}{ Dependencia Alta } & $0,8 \%(n=18)$ \\
\hline \multicolumn{3}{|c|}{ Consumo Alcohol (AUDIT) } \\
\hline \multirow{4}{*}{$\begin{array}{l}\text { Cantidad y } \\
\text { Frecuencia }\end{array}$} & No Consume & $44,9 \%(n=958)$ \\
\hline & Escaso Consumo & $25,4 \%(n=541)$ \\
\hline & Consumo Medio & $23,1 \%(n=494)$ \\
\hline & Consumo Alto & $6,6 \%(n=141)$ \\
\hline \multirow{4}{*}{ Dependencia } & No Dependencia & $79,4 \%(n=1.695)$ \\
\hline & Dependencia Baja & $13,4 \%(n=285)$ \\
\hline & Dependencia Media & $5,8 \%(n=124)$ \\
\hline & Dependencia Alta & $1,4 \%(n=30)$ \\
\hline \multirow{4}{*}{$\begin{array}{l}\text { Consumo } \\
\text { Dañino }\end{array}$} & No Daño & $65,3 \%(n=1.394)$ \\
\hline & Daño Escaso & $17,8 \%(n=379)$ \\
\hline & $\begin{array}{c}\text { A Veces provoca } \\
\text { Daño }\end{array}$ & $11,8 \%(n=251)$ \\
\hline & Siempre Daño & $5,2 \%(n=110)$ \\
\hline \multicolumn{3}{|c|}{ AF Regular } \\
\hline & SI & $67,1 \%(n=1.431)$ \\
\hline & NO & $32,9 \%(n=703)$ \\
\hline \multicolumn{3}{|c|}{ Tipo de Deporte } \\
\hline Deport & Individuales & $14,4 \%(n=307)$ \\
\hline Depor & s Colectivos & $27,2 \%(n=581)$ \\
\hline Activida & s de Gimnasio & $4,3 \%(n=91)$ \\
\hline & tros & $21,2 \%(n=452)$ \\
\hline \multicolumn{2}{|c|}{ No Práctica } & $32,9 \%(n=703)$ \\
\hline \multicolumn{3}{|c|}{ Nivel Federado } \\
\hline & SI & $21,9 \%(n=467)$ \\
\hline & NO & $78,1 \%(n=1.667)$ \\
\hline \multicolumn{3}{|c|}{ Padres AF Regular } \\
\hline & SI & $51,8 \%(n=1.105)$ \\
\hline & NO & $48,2 \%(n=1.029)$ \\
\hline
\end{tabular}

Tabla II. Relación del tabaco y el consumo de alcohol según práctica de A.F.

\begin{tabular}{cc}
\hline Parámetros & $\boldsymbol{X}^{2}$ \\
\hline Tabaco & $\mathrm{p}=.035$ \\
\hline Cantidad y Frecuencia de Alcohol & $\mathrm{p}=.975$ \\
\hline Dependencia del Alcohol & $\mathrm{p}=.113$ \\
\hline Daño por Alcohol & $\mathrm{p}=.380$ \\
\hline
\end{tabular}

Tabla III. Relación de la modalidad deportiva y estado federativo. $\left(p=.000^{* *}\right)$

\begin{tabular}{|c|c|c|c|c|}
\hline \multirow{2}{*}{\multicolumn{2}{|c|}{$\begin{array}{c}\text { Tipo de Deporte } \\
\text { Si }\end{array}$}} & \multicolumn{2}{|c|}{ ¿Estás federado? } & \multirow{2}{*}{ Total } \\
\hline & & No & & \\
\hline \multirow{3}{*}{$\begin{array}{c}\text { Deportes } \\
\text { Individuales }\end{array}$} & Recuento & 104 & 203 & 307 \\
\hline & \% TipoDeporte & $33,9 \%$ & $66,1 \%$ & $100,0 \%$ \\
\hline & $\begin{array}{l}\text { \%¿Estás } \\
\text { Federado? }\end{array}$ & $22,3 \%$ & $21,1 \%$ & $21,5 \%$ \\
\hline \multirow{3}{*}{$\begin{array}{l}\text { Deportes } \\
\text { Colectivos }\end{array}$} & Recuento & 241 & 340 & 581 \\
\hline & \% Tipo Deporte & $41,5 \%$ & $58,5 \%$ & $100,0 \%$ \\
\hline & $\begin{array}{l}\% \text { ¿Estás } \\
\text { Federado? }\end{array}$ & $51,6 \%$ & $35,3 \%$ & $40,6 \%$ \\
\hline \multirow{3}{*}{$\begin{array}{c}\text { Actividades } \\
\text { Gimnasio }\end{array}$} & Recuento & 20 & 71 & 91 \\
\hline & \% Tipo Deporte & $22,0 \%$ & $78,0 \%$ & $100,0 \%$ \\
\hline & $\begin{array}{l}\% \text { ¿Estás } \\
\text { Federado? }\end{array}$ & $4,3 \%$ & $7,4 \%$ & $6,4 \%$ \\
\hline \multirow{3}{*}{ Otros } & Recuento & 102 & 350 & 452 \\
\hline & \% Tipo Deporte & $22,6 \%$ & $77,4 \%$ & $100,0 \%$ \\
\hline & $\begin{array}{l}\% \text { ¿Estás } \\
\text { Federado? }\end{array}$ & $21,8 \%$ & $36,3 \%$ & $31,6 \%$ \\
\hline \multirow{3}{*}{ Total } & Recuento & 467 & 964 & 1431 \\
\hline & \% Tipo Deporte & $32,6 \%$ & $67,4 \%$ & $100,0 \%$ \\
\hline & $\begin{array}{l}\% \text { ¿Estás } \\
\text { Federado? }\end{array}$ & $100,0 \%$ & $100,0 \%$ & $100,0 \%$ \\
\hline
\end{tabular}

En cuanto al consumo o ingesta de tabaco y alcohol y la práctica regular de actividad física como primer predictor los resultados reportaron diferencias estadísticamente significativas en cuanto a la variable "Tabaco" $(p=.035)$, propiciada porque los sujetos que no practican actividad física son más fumadores que los practicantes, al compararlos con el "Consumo de Alcohol" en las tres categorías no se reportaron diferencias $(p \geq .050)$, como se ve en la siguiente tabla (Tabla II):

En lo concerniente al análisis de los índices de práctica de Actividad Física en cuanto al tipo de modalidad deportiva, los datos mostraron relación estadísticamente significa- 
Tabla IV. Relación de práctica de A.F con realización de A.F por parte de padres. $\left(p=.000^{* *}\right)$

\begin{tabular}{ccccc}
\hline \multirow{2}{*}{$\begin{array}{c}\text { Practica de A.F } \\
\text { Si }\end{array}$} & \multicolumn{2}{c}{$\begin{array}{c}\text { Padres } \\
\text { practican A.F }\end{array}$} & \multirow{2}{*}{ Total } \\
\cline { 2 - 4 } & & No & \\
\hline \multirow{3}{*}{ SI } & Recuento & 839 & 592 & 1431 \\
\cline { 2 - 4 } & $\%$ Práctica A.F & $58,6 \%$ & $41,4 \%$ & $100,0 \%$ \\
\cline { 2 - 4 } & $\%$ ¿Padres A.F.? & $75,9 \%$ & $57,5 \%$ & $67,1 \%$ \\
\hline \multirow{3}{*}{ NO } & Recuento & 266 & 437 & 703 \\
\cline { 2 - 4 } & $\%$ Práctica A.F & $37,8 \%$ & $62,2 \%$ & $100,0 \%$ \\
\cline { 2 - 4 } & $\%$ ¿Padres A.F.? & $24,1 \%$ & $42,5 \%$ & $32,9 \%$ \\
\hline \multirow{3}{*}{ Total } & Recuento & 1105 & 1029 & 2134 \\
\cline { 2 - 4 } & $\%$ Práctica A.F & $51,8 \%$ & $48,2 \%$ & $100,0 \%$ \\
\cline { 2 - 4 } & $\%$ ¿Padres A.F.? & $100,0 \%$ & $100,0 \%$ & $100,0 \%$ \\
\hline
\end{tabular}

tiva $\left(p \leq .05^{* *}\right)$, propiciada por lo estudiantes que no practican regularmente deporte, si excluimos a estos del análisis los resultados deparan también falta de asociación establecida porque los adolescentes se decantan en su mayoría por deportes colectivos $(n=581 ; 40,6 \%)$ y otros $(n=452 ; 31,6 \%)$, frente a los que se inclinan más por actividades individuales $(n=307 ; 21,4 \%)$ o de gimnasio $(n=91 ; 6,4 \%)$.

Del mismo modo al estudiar en que modalidad deportiva encontramos mayor número de fichas federativas los resultados nos muestran diferencias estadísticamente significativas $\left(p \leq .05^{* *}\right)$, propiciadas porque en deportes de tipo colectivo $(41,5 \%)$ e individual $(33,9 \%)$ los adolescentes se encuentran más inscritos que en actividades de gimnasio y otros con valores en ambos casos del $22 \%$.

Los resultados concernientes en cuanto a la posible relación que tendría la realización de A.F de una forma regular en cuanto a que alguno de los progenitores también la desarrollasen habitualmente generó discrepancia estadística $\left(p \leq .05^{\star *}\right)$, motivada porque en aquellos adolescentes que si practicaban A.F. de manera regular los padres también lo hacían en un $58,6 \%$ de las veces mientras que a la inversa en jóvenes no practicantes, los padres no los hacían en un $62,2 \%$ de las veces.

\section{Discusión}

Los datos reportados nos indicaron que la mayoría de adolescentes analizados no consumían tabaco y muy pocos de ellos ingerían alcohol de forma habitual; en esta línea hemos hallado estudios previos como los de Rojas, Fleiz, Medina, Moron, y Doménech (1999); Landeroy Villareal-González (2007); López-García, Esparza, Guzmán, Alonso, y Rodríguez (2009); Salamó, Gras y Font (2010) o Carballo y cols. (2013), donde los porcentajes de ingesta de alcohol en diversos contextos geográficos son semejantes. Sin embargo, se constata que tres de cada diez estudiantes sí lo consumían de forma esporádica (fines de semana) o habitual. Estos jóvenes comienzan a realizar salidas nocturnas los fines de semana y, como exponen Martínez y Villar (2004) y Ruiz-Ruiseño y cols. (2011) una de las causas influyentes en el mayor consumo de alcohol y tabaco en adolescentes españoles puede deberse al fenómeno "botellón"1asentado desde hace décadas en nuestro país.

La principal explicación de los bajos porcentajes de consumo de ambas sustancias, es que la muestra estudiada es relativamente joven para presentar valores de consumo habituales altos, siendo la edad de 16 en adelante la que presenta porcentajes mayores en este campo. De ahí que los resultados deban interpretarse con cautela. Estos resultados podrían deberse a que en estas edades es más habitual beber de forma esporádica, como los fines de semana y días festivos, que diariamente. De ahí que la mayoría de estudios muestren porcentajes elevados en cuanto a la ingesta de alcohol de forma eventual y, por el contrario, bajos en lo que a dependencia se refiere.En cualquier caso, se hace imprescindible la transmisión de hábitos familiares saludables por parte de la familia desde edades tempranas, ya que las probabilidades de aumento en consumo progresa conforme se incrementa la edad (Gómez, Fernández, Romero, \& Luengo, 2008).

1 Es un fenómeno surgido entre la juventud de España en la década de los 90 , consistente en que los jóvenes organizan las noches de los fines de semana en espacios públicos al aire libre, para ello compran alcohol y otras bebidas y las consumen en grupo (Calafat \& cols., 2005). 
En cuanto a la actitud violenta tras la ingesta de alcohol en el trabajo de Pascual, Reig, Fontoba, y García (2011), se estudió la relación entre alcohol y violencia; extrayéndose cifras bastante similares a los reportados por nuestra muestra, así entre los consumidores una tercera parte declaró mostrar actitudes dañinas tras la ingesta de estos productos, como reyertas, conducción de algún vehículo y actos de comportamiento inadecuados; por el contrario debemos destacar que la mayoría mantenían conductas adecuadas al igual que afirmaban Meneses, Markez, Romo, Uroz, Rua, y Laespada (2013), entendemos que este hecho se debe a que la cantidad de bebida tomada no es desmesurada hasta el punto de provocar actos improcedentes en la persona. Idea apoyada a su vez por la baja edad de la mayoría del alumnado encuestado, lo cual nos incita a sospechar que a menos edad, menos cantidad de alcohol se ingiere y por ende, más concordancia en los actos se tiene.

Con respecto a la práctica regular de Actividad Física tres cuartas partes de los adolescentes granadinos la realizaban de forma sistemática, resultados similares encontraron Yuste, López, López, García, y García (2008) en la región de Murcia, Martínez-Gómezy cols. (2009) en Madrid o Beltrán-Carrillo, Devís-Devís y Peiró-Velert (2012) en la Comunidad Valenciana que señalan dos extremos, (actividad del 52,2\% e inactividad del $47,8 \%$ ) y destacar los resultados similares de Riddoch y cols. (2004), en adolescentes de Portugal, Estonia, Dinamarca y Noruega.

Por el contrario, Janssen y cols. (2005) investigaron el nivel de práctica de AF en 34 países europeos, donde descubrió que sólo el 25,4\% llevaba a cabo una práctica física adecuada.El hecho de haber obtenido este tipo de resultados estimamos se debe a la gran cantidad de actividades físicas ofertadas por los ayuntamientos y centros escolares hoy día. Al mismo tiempo, nuestro estudio cuenta con un buen porcentaje de muestra en zona costera, lo cual invita más a la práctica deportiva debido al buen clima.

Los resultados arrojaron que los sujetos que no practican actividad física son más fumadores que los practicantes; estos datos concuerdan con los hallados por Ruiz-Ruisueño y cols. (2011), donde compara la relación entre actividad física y consumo de drogas con una muestra de adolescentes españoles y mexicanos. Citado autor, afirma que el adolescente español que es activo, tiene una menor predisposición a consumir sustancias nocivas. Por el contrario, en la población mexicana, la práctica de actividad física no se vinculó con una menor ingesta de drogas. Sin embargo, pocos son los estudios que evidencian que la práctica de actividad física pueda provocar hábitos no saludables (Rainey, McKeown, Sargent, \& Valois, 1996).

Pensamos que los resultados encontrados en la relación tabaco y actividad física, de deben a que la ingesta de nicotina repercute negativamente en el rendimiento deportivo. Por el contrario, el hecho de consumir alcohol, no es acusado, en tanta medida, como el tabaco en el rendimiento deportivo por parte de los alumnos, los cuales llevan a cabo un consumo más esporádico, concretamente de fin de semana, y como elemento social.
La mayoría de los adolescentes practican deportes de tipo colectivo, especialmente el futbol, proporciones muy similares son las mostradas en el estudio llevado a cabo por Ruiz y cols. (2001) en la población almeriense, donde el $44,8 \%$ se decantaba por deportes colectivos frente al $33,7 \%$ que prefería modalidades individuales. Por el contrario, García (2006) en su amplia investigación sobre los deportes más practicados desde 1980-2005, descubrió que la natación había sido la modalidad más practicada en estos veinticinco años, posicionándose por encima de deportes como el fútbol, voleibol y baloncesto, tradicionalmente predominantes en nuestro país, entendemos que el rango de edad empleado por el autor era diverso. Nuestra explicación a tales resultados, la atribuimos a que los deportes colectivos ofrecen mayor posibilidad de diversión y relaciones sociales entre amigos, lo cual lo hace más entretenido y atractivo en estas edades.

En nuestro trabajo se encuentran cifras semejantes a las que nos deparan Revuelta y Esnaola (2011) en adolescentes procedentes del País Vasco y Cantabria, que nos indicanque la influencia de los padres es decisiva en la práctica deportiva por parte de los hijos, habiendo un porcentaje alto de niños practicantes de actividad física cuyos padres estaban inmiscuidos en ámbitos deportivos. También Fredricks y Eccles (2004) hablan de la importancia del contacto de la persona con el deporte desde edades tempranas, lo cual transmite cantidad de valores de forma directa e indirecta. Los resultados encontrados en la presente investigación guardan relación con lo hallado por la mayoría de los investigadores. En este sentido, pensamos que el hecho de que los niños tengan una familia que practique deporte y actividad física, repercute positivamente en que éstos desarrollen prácticas similares durante su adolescencia.

Este trabajo complementa a numerosos estudios realizados en poblaciones adolescentes en relación a los hábitos saludables, aportando nuevos datos en una población como es la de Segundo Ciclo de Educación Secundaria Obligatoria que constituye una de las etapas cronológicas clave para el desarrollo social del adolescente; asimismo entendemos que las principales limitaciones que nos invitan a interpretar los datos con cierta cautela ha sido la de realizar un estudio de carácter transversal no permitiéndonos establecer relaciones causales y no haber profundizado en la procedencia real de estos participantes, que si bien si se considerarán en futuros proyectos educativos a realizar.

\section{Conclusiones}

Los estudiantes jóvenes analizados son en su mayoría no fumadores y consumen alcohol esporádicamente (fines de semana); asimismo la práctica de actividad física regular es perceptible en siete de cada diez adolescentes siendo la modalidad de deporte colectivo la más frecuentada y dos de cada diez se encuentran federados.En la unidad familiar, más de la mitad de los padres/madres habitualmente hacen actividad físico-deportiva, igualmente destacar que 
entre los jóvenes practicantes se encontró asociación con el consumo de tabaco, el tipo de actividad practicada y nivel de práctica en los padres.

También debemos indicar que los adolescentes que practican actividad física de una forma regular vienen inducidos por el no consumo de sustancias nocivas así como por la influencia de parámetros familiares, realizando en mayor medida deportes de tipo colectivo y estando federados en ellos, asimismo puntualizar que las tres hipótesis planteadas se cumplen.

Estos hallazgos respaldan la necesidad de seguir profundizando en la investigación de hábitos saludables y de personalidad en los adolescentes, con el objetivo de detectar la etapa clave donde se generan los comportamientos nocivos que afectan a los jóvenes y que repercuten negativamente en edades superiores. Parece incuestionable la necesidad de determinar los factores antecedentes así como elaborar programas de actividad física encaminados a evitar el número de abandono de la realización de ejercicio de forma habitual.

\section{Referencias}

Abarca-Sos, A., Zaragoza, J., Generelo, E., \& Julián, J. A. (2010). Comportamientos sedentarios y patrones de actividad física en adolescentes. Revista Internacional de Medicina y Ciencias de la Actividad Física y del Deporte, 40(39), 410-427.

Ariza, C., Nebot, M., Villalbí, J.R., Díez, E., Tomás, Z.,\&Valmayor, S. (2003). Tendencias en el consumo de tabaco, alcohol y cannabis de los escolares de Barcelona (1987-1999). Gaceta Sanitaria,17, 190-195.

Beltrán-Carrillo, V.J., Devís-Devís, J., \& Peiró-Velert, C. (2012). Actividad física en adolescentes de la comunidad valenciana. Revista Internacional de Medicina y Ciencias de la Actividad Física y del Deporte, 12(45), 123-137.

Buelga, S., Musitu, G., \& Murgui, S. (2009). Relaciones entre la reputación social y la agresión relacional en la adolescencia. International Journal of Clinical and Health Psychology, 9, 127141.

Calafat, A., Juan, M., Becoña, E., Castillo, A., Fernández, C., Franco, M., Pereiro, C., \& Ros, M. (2005). El consumo de alcohol en la lógica del botellón. Adicciones, 17(3), 193-200.

Carballo, J.L., Marín, M., Jáuregui, V., García, G., Espada, J., Orgilés, M.,\& Piqueras, J.A. (2013). Consumo excesivo de alcohol y rendimiento cognitivo en estudiantes de secundaria de la provincia de Alicante. Health and addictions: Salud y drogas, 13(2), 157163.

Cava, M.J., Murgui, S., \& Musitu, G. (2008). Diferencias en factores de protección del consumo de sustancias en la adolescencia temprana y media. Psicothema, 20, 389-395.
Cortés, M.T., Espejo, B., Giménez, J.A., Luque, L., Gómez, R., \& Motos, P. (2011). Creencias asociadas al consumo intensivo de alcohol entre adolescentes. Health and addictions: Salud y Drogas, 11(2), 179-202.

Elzo, J. (2010). ¿Hay un modelo mediterráneo de consumo de alcohol? Hablemos de alcohol. Madrid: Entinema.

Fredricks, J. A. \&Eccles, J. S. (2004).Parental Influences on Youth Involvement in Sports. Morgantown, WV: M. Weiss.

García, M. (2006). Veinticinco años de análisis del comportamiento deportivo de la población española (1980-2005). Revista Internacional de Sociología, 64(44), 15-38.

Giró, J. (2007). Adolescentes, ocio y consumo de alcohol. Madrid: Entinema.

Gómez, J.A., Fernández, N., Romero, E.,\& Luengo, A. (2008). El botellón y el consumo de alcohol y otras drogas en la juventud. Psicothema, 20(2), 211-217.

Heatherton, T., Kozlowski, L., Frecker R., \& Fagerström, K.O. (1991). The Fagerström Test of Nicotine Dependence: a revision of the Fagerström Tolerance Questionnaire. British Journal of Addiction, 86(9), 1119-1127.

Henry, K., Slater, M., \& Oetting, E. (2005). Alcohol Use in Early Adolescence: The Effect of Changes in Risk Taking, Perceived Harm and Friends' Alcohol Use. Journal of Studies on Alcohol and Drugs, 66, 275-283.

Janssen, I., Katzmarzyk, P.T., Boyce, W.F., Vereecken, C., Mulvihill, C., Roberts, C. Currie, C., \& Pickett, W. (2005). Comparison of overweight and obesity prevalence in school-aged youth from 34 countries and their relationships with physical activity and dietary patterns. Obesity Review, 6(2), 123-132.

Jiménez, E., Musitu, G., \& Murgui, S. (2008). Funcionamiento familiar y consumo de sustancias en adolescentes: el rol mediador de la autoestima. Int J Clin Health Psychol, 8(1), 139-151.

Krekoukia, M., Nassis, G. P., Psarra, G., Skenderi, K., Chrousos, G. P., \& Sidosis, L. S. (2007). Elevated total and central adiposity and low physical activity are associated with insulin resistance in children. Metabolism, 56, 206-213.

Landero, R. \& Villarreal-González, M.E. (2007). Consumo de alcohol en estudiantes en relación con el consumo familiar y de los amigos. Psicología y Salud, 17(1), 17-23.

Londoño, C. (2010). Resistencia de la presión de grupo, creencias acerca del consumo y consumo de alcohol en universitarios. Anales de Psicología, 26(1), 27-33.

López-García, K.S., Esparza, S.E., Guzmán, F.R., Alonso, M.M., \& Rodríguez, L. (2009). Expectativas del consumo de alcohol y 
tabaco en adolescentes escolares. Revista Científica Electrónica de Psicología, 7, 112-126.

Luengo, C. (2007). Actividad físico-deportiva extraescolar en alumnos de primaria. Revista Internacional de Medicina y Ciencias de la Actividad Física y el Deporte, 7(27), 174-184.

Martínez, G. \& Villar, M. (2004). Estudio descriptivo del uso de drogas en adolescentes de educación media superior de Cd. de Monterrey N.L. Revista Latinoamericana Enfermagem, 12, 391 -397.

Martínez-Gómez, D., Welk, G. J., Calle, M. E., Marcos, A., \& Veiga, O. L. (2009). Preliminary evidence of physical activity levels measured by accelerometer in Spanish adolescents: the AFINOS Study. Nutrición Hospitalaria, 24 (2), 226-232.

Maurage, P., Joassin, F., Speth, A., Modave, J., Philippot, P., \& Campanella, S. (2012). Cerebral effects of binge drinking: Respective influences of global alcohol intake and consumption pattern. Clinical Neurophysiology, 123, 892-901.

Meneses, C., Markez, I., Romo, N., Uroz, J., Rua, A., \& Laespada, T. (2013). Diferencias de género en el consumo diario de tabaco e intensivo de alcohol en adolescentes latinoamericanos en tres áreas españolas (Andalucía, Madrid y País Vasco). Revista de la Asociación Española de Neuropsiquiatría, 33(119), 525-535.

Musitu, G. \& Pons, J. (2010). Adolescencia y alcohol: Buscando significados en la persona, la familia y la sociedad. En J. Elzo (Org.), Hablemos de alcohol: Por un nuevo paradigma en el beber adolescente (137-170). Madrid: Entimema.

Nebot, M., Tomás, Z., Ariza, C., Valmayor, S., López, M.J., \& Juárez, O. (2004). Factores asociados al inicio del tabaquismo: seguimiento a los 3 años de una cohorte de escolares. Archivos de Bronconeumología, 40, 495-501.

Nerín, I., Beamonte, A., Gargallo, P., Jiménez-Muro, A.,\& Marqueta, A. (2007). Ganancia ponderal al dejar de fumar y su relación con la ansiedad. Archivos de Bronconeumología, 43, 9-15.

Organización Mundial de la Salud. (2010). Recomendaciones mundiales sobre actividad física para la salud. Ginebra: WHO.

Oviedo, G., Sánchez, J., Castro, R., Calvo, M., Sevilla, J.C., Iglesias, A., \& Guerra, M. (2013). Niveles de actividad física en población adolescente: estudio de caso. Retos. Nuevas tendencias en Educación Física, Deporte y Recreación, 23, 43-47.

Pascual, F., Reig, M., Fontoba, J., \& García, A. (2011). Alcohol y violencia. Health and Addictions: Salud y Drogas, 11(1), 71-94.

Plan Nacional sobre Drogas (2013). Encuesta sobre alcohol y drogas en población general en España. Edades 2011-2012. Madrid: Delegación del Gobierno para el Plan Nacional sobre Drogas.

Pons, J. (1998). El modelado familiar y el papel educativo de los padres en la etiología del consumo de alcohol en los adolescentes. Revista Española de Salud Pública, 72, 251-266.

Rainey, C. J., McKeown, R.E., Sargent, R.G., \& Valois, R.F. (1996). Patterns of tobacco and alcohol use among sedentary, exercising, nonathletic, and athletic youth.JournalSchoolHealth.66(1), 27-32.

Revuelta, L. \& Esnaola, I. (2011). Clima familiar deportivo y autoconcepto físico en la adolescencia. European Journal of Education and Psychology, 4(1), 19-31.

Riddoch, C. J., Bo Andersen, L., Wedderkopp, N., Harro, M., KlassonHeggebø, L., Sardinha, L. B., Cooper, A.R., \& Ekelund, U. (2004). Physical activity levels and patterns of 9- and 15-yr-old European children. Medicine and Sciences in Sports and Exercise, 36(1), 8692.

Rojas, E., Fleiz, C., Medina, M., Morón, M., \& Doménech, M. (1999).Consumo de alcohol y drogas en estudiantes de Pachuca (Hidalgo). Revista de Salud Pública de México, 41(4), 297-308.

Rubio, G. (1998). Validación de la prueba para la identificación de trastornos por el uso de alcohol (AUDIT) en Atención Primaria. Revista Clínica Especializada, 198, 11-14.

Ruiz, F., García, M. E.,\& Hernández, A. I. (2001). Comportamientos de actividades físico-deportivas de tiempo libre del alumnado almeriense de enseñanza secundaria post obligatoria. Revista Motricidad,7, 113- 143.

Ruiz-Juan, F. \& Ruiz-Risueño, J. (2011). Variables predictoras de consumo de alcohol entre adolescentes españoles. Anales de Psicología, 27(2), 350-359.

Ruiz-Ruiseño, J., Ruiz-Juan, F.,\& Zamarripa J.I. (2011). Alcohol y tabaco en adolescentes españoles y mexicanos y su relación con la actividad físico-deportiva y la familia. Revista Panamericana Salud Publica, 31(3), 211-220.

Salamó, A., Gras, M.E.,\& Font, S. (2010). Patrones de consumo de alcohol en la adolescencia. Psicothema, 22(2), 189-195.

Santos, J., Muñoz, P. Juez, J., \& Cortiñas, A. (2003). Diseño de Encuestas para estudios de mercado. Técnicas de muestreo y análisis multivariante. Madrid: Editorial Ramón Areces.

Saunders, J., Aasland, O., Babor, T., De la Fuente, J., \& Grant, M. (1993).Development of the Alcohol Use Disorders Identification Test (AUDIT): Who collaborative Project on early detection of persons with harmful alcohol consumption-II. Addiction, 88, 791804.

Villareal-González, M.E. (2009). Un modelo estructural del consumo de drogas y conducta violenta en adolescentes escolarizados. Tese de doutorado, Universidad Autónoma de Nuevo León, México. 
Villarreal, M. E., Sánchez-Sosa, J. C., Musitu, G., \& Varela, R. (2012). El consumo de alcohol en adolescentes escolarizados: propuesta de un modelo sociocomunitario. Intervención Psicosocial, 19(3), 253-264.

Villarreal-González, M.E., Sánchez, J.C., \& Musitu, G. (2013). Análisis psicosocial del consumo de alcohol en adolescentes mexicanos. Universitas Psychologica, 12(3), 857-873.
Woolfolk, A. (2008). Educational Psychology. Boston: Allyn y Bacon.

Yuste, J.L., López, P.A., López, F.J., García, J.V.,\&García, J.J. (2008). En Anais do IV Congreso Internacional y XXV Nacional de Educación Física. Palacio de Exposiciones y Congresos de Córdoba.

\section{Sobre os autores}

José Ignacio Álvaro González (nachoalvarog@hotmail.com)

Licenciado en Ciencias de la Actividad Física y el Deporte

Félix Zurita Ortega (felixzo@ugr.es)

Profesor Doctor del Área de Corporal de la Universidad de Granada (España)

Virginia Viciana Garófano (vviciana@ugr.es)

Profesor Doctor del Área de Corporal de la Universidad de Granada (España)

Asunción Martínez Martínez(asunciomm@ugr.es)

Doctora en Educación. Investigadora del Grupo HUM-238 de la Universidad de Granada (España).

Susana García Sánchez (s_garcia_s@hotmail.com)

Maestra Especialista en Primaria.

Manuel Estévez Díaz (manoloestevez@hotmail.com\}

Doctor y Licenciado en Ciencias de la Actividad Física y el Deporte 